\title{
Du dessin au dessein des plantes sauvages
}

\section{Florence Brunois}

\section{(2) OpenEdition}

\section{Journals}

\section{Édition électronique}

URL : http://journals.openedition.org/jso/1359

DOI : 10.4000/jso.1359

ISSN : $1760-7256$

\section{Éditeur}

Société des océanistes

\section{Édition imprimée}

Date de publication : 1 décembre 2002

Pagination : 23-38

ISSN : 0300-953x

\section{Référence électronique}

Florence Brunois, «Du dessin au dessein des plantes sauvages », Journal de la Société des Océanistes [En ligne], 114-115 | Année 2002, mis en ligne le 26 mai 2008, consulté le 22 mars 2021. URL : http:// journals.openedition.org/jso/1359; DOI : https://doi.org/10.4000/jso.1359

\section{(c) (i) (9)}

Journal de la société des océanistes est mis à disposition selon les termes de la Licence Creative Commons Attribution - Pas d'Utilisation Commerciale - Pas de Modification 4.0 International. 


\title{
Du dessin au dessein des plantes sauvages
}

\author{
par
}

Florence BRUNOIS *

\section{RÉSUMÉ}

Relatant son enquête ethnobotanique menée auprès de la société kasua (PNG), l'auteur illustre le rôle que prêtait Jacques Barrau au dessin botanique comme outil ethnographique pour appréhender le monde vivant mais aussi la place de l'homme dans le monde vivant.

MotS-CLÉS : dessin botanique, outil ethnographique, usages de la flore, logiques interactives, personnalité végétale.

«Entrez», m'invita la voix de Jacques Barrau. « Entrez, entrez!», dut-il répéter pour que j'ose enfin franchir la porte de son bureau. Pourquoi mon hésitation? Aujourd'hui encore, je m'interroge. L'éminent chercheur et professeur avec qui j'avais rendez-vous ne se montrait pourtant pas homme intimidant avec ses étudiants. Bien au contraire. Que ce soit au cours du séminaire d'ethnoscience qu'il organisait à la salle Chevalier, au croisement d'une rue, ou encore dans ce fameux bureau, Jacques Barrau se révélait un professeur accessible délivrant avec générosité ses immenses connaissances de l'ethnobotanique océanienne, s'enthousiasmant sur votre propre recherche aussi balbutiante qu'elle puisse être avant la réalisation du terrain. Depuis 1991, sa gentillesse m'était devenue familière et l'intérêt qu'il portait à mon projet de thèse m'était devenu précieux. En me faisant confiance, Jacques Barrau me donnait confiance.

Ainsi, rien a priori ne pouvait expliquer la retenue qui m'anima ce jour de l'année 1994. Ou peut-être alors, et finalement, l'objet de mon

\section{ABSTRACT}

Relating her ethnobotanical inquiry conducted among the Kasua of Papua New Guinea, the author illustrates the role that Jacques Barrau confered to the botanical drawings as an ethnographical tool to apprehend the living world as well as human's position within the living world.

KEYWORDS : botanical drawings, ethnographical tool, flora's usage, interactives logics, plant's personality.

rendez-vous qui ne ressemblait pas aux autres prétextes passés me conduisant jusqu'à lui. En effet, il n'était pas question de l'interroger sur le palmier sagoutier Metroxylon sp., de lui demander le nombre de plantes utiles rencontrées en Océanie, ni de lui solliciter une lettre de recommandation. Non, je venais juste lui dire au revoir. Ayant reçu mon visa de recherche après trois longues années d'attente, j'allais enfin partir en Papouasie Nouvelle-Guinée pour étudier les relations qu'entretient la société Kasua du Mont Bosavi avec son univers forestier tropical. Autrement dit, j'allais enfin me réaliser m'éprouver - en tant qu'ethnographe. Et c'était bien la perspective de lui annoncer - il en sera le premier informé - qui, je crois, eut le pouvoir de m'intimider, me rendant soudainement sourde à son invitation d'entrer dans son bureau. Jacques Barrau insista, bien heureusement ! Notre entrevue fut non seulement la dernière que nous ayons

* CREDO-MAP UMR 6574 EHESS-CNRS, Université de Provence. Je voudrais remercier très chaleureusement Alice Peeters pour avoir prêté à l'occasion de cet hommage les superbes reproductions des dessins de Jacques Barrau. 
eue, mais aussi, celle qui eut le plus d'incidence sur ma recherche ethnographique. Aussi, tout naturellement, c'est sur cette ultime rencontre et ses implications ethnologiques sur lesquelles je voudrais aujourd'hui revenir pour honorer sa mémoire.

\section{La découverte d'un outil ethnographique original : le dessin}

Constatant mon émotion quelque peu paralysante, le professeur Jacques Barrau inversa judicieusement les rôles. Il m'assaillit de questions de tout ordre : sur mon départ, ses conditions, les médicaments composant ma pharmacie, etc... Il s'inquiétait, semble-t-il, mais je le rassurai en l'informant que mon compagnon partait avec moi. « Il est ethnologue, lui aussi, alors ? » me demanda-t-il ? « Non, non », lui répondis-je, « il est artiste peintre ». L'écoute de ces derniers mots l'enjoua soudainement. Il se leva, me fit signe de le suivre, et me mena devant une de ses œuvres picturales - omniprésentes et pourtant si discrètes : étaient-elles seulement encadrées ? - suspendue à l'un des murs de son bureau. Jacques Barrau, peintre naturaliste, était un homme humble. Il semblait nourrir cette passion silencieusement, comme on cultive son jardin secret, même s'il est vrai qu'il était parmi les rares ethnologues illustrant ses écrits scientifiques de reproductions graphiques. Reste que les planches qu'il me montra alors s'en distinguaient étonnamment. Non qu'elles étaient abstraites, mais, au réalisme rigoureux de son coup de crayon s'ajoutait la magie des couleurs, la magie de la peinture. La fleur, le fruit ou l'animal, loin de participer à une nature morte, évoquait la vie, leur vie ; évoquait, de manière tout aussi éclatante, le propre goût de la vie et de la nature de leur peintre (Figs 1 et 2). Sans le vouloir, Jacques Barrau me démontrait que la science pouvait s'allier amicalement à l'art. Ce constat, qui me réjouissait, m'incita à lui révéler cette amusante anecdote : nous nous rendions, Haudricourt et moi-même, au séminaire du professeur Hagège et nous nous arrêtâmes devant une fresque située à la Sorbonne. Cet arrêt avait été voulu par Haudricourt avec qui je m'entretenais souvent sur l'art. Il souhaitait me montrer, en fait, sur quoi reposait son désaccord, son manque d'intérêt et de considération pour la création artistique. S'approchant de l'œuvre, il prit sa canne énergiquement et la tendit vers une plante reproduite de manière très réaliste: « vous voyez cette plante (qu'il me nomma bien sûr mais dont j'ai oublié le nom), elle n'existait pas à l'époque de cette scène historique reproduite ici. C'est un trompe-savant!». Jacques Barrau se mit à rire, reconnaissant sûrement dans ce petit récit la personnalité de son ami. Cependant, cette réflexion haudricourtienne ne concernait pas vraiment son œuvre qui nous intéresse ici : le dessin. Jacques Barrau abordait sa pratique du graphisme non pas comme une technique d'expression artistique, mais comme un véritable outil de recherche scientifique. « Oui », affirmait-il, « le dessin est, pour moi, un outil ethnographique ». Et en tant que telle, son intention était incontestablement de rendre compte de la dimension humaine, de rendre compte de la place de l'homme dans la nature. Pour s'en convaincre, il suffit d'ailleurs d'observer les croquis jalonnant ses ouvrages. Un exemple révélateur nous est ainsi offert à la page 160 de sa thèse où, plutôt que d'évaluer la taille du fruit du Pandanus tectorius en dessinant à ses côtés une échelle de mesure centimétrique, il préféra à cette évaluation abstraite, celle d'un humain - tout aussi abstrait —, tenant dans ses mains le fruit en question. Ses dessins des techniques horticoles ou de l'exploitation du palmier sagoutier témoignent de cette même volonté d'appréhender l'homme dans/avec son milieu, en mettant l'accent sur sa tenue corporelle, autrement dit, en mettant l'accent sur l'espace et la forme que le corps humain occupe lorsqu'il est en interaction avec d'autres êtres vivants. Et si la photographie offre des possibilités sensiblement similaires avec en outre plus de facilités, Jacques Barrau lui préférait assurément le dessin car, disait-il, et comme le trahissent ses écrits ethnobotaniques, «par le geste de reproduire manuellement ce qu'il observait, il s'en imprégnait, plus attentif à ce qu'il étudiait, plus en harmonie ». Son œil était ainsi plus objectif que tous les objectifs photographiques : «il voyait tous les détails, même ceux des ombres portées des palmes qui offrent une extraordinaire diversité de tonalités!» (Alice Peeters, com. pers). Par ailleurs, précisait-il, "ses dessins lui permettaient de prolonger sa réflexion et son enquête auprès des gens ». En effet, à la différence de la plante ou d'une technique dont l'observation est éphémère, ou encore d'une photo dont il faut attendre le développement, ses reproductions graphiques étaient immédiatement disponibles ; elles lui étaient toujours accessibles où qu'il se trouve. Tous ces usages dont il investissait l'outil graphique expliquaient certainement son souci de l'exactitude scrupuleuse dans le trait, conférant à ses dessins, au-delà de leur qualité artistique indéniable, leur valeur scientifique : naturaliste et ethnographique. 
Je le quittai sur ce bel échange, enrichie de sa manière originale et croquante d'appréhender la diversité du monde vivant, d'appréhender la place de l'homme dans le monde vivant, deux applications fondamentales et inattendues du dessin que j'éprouvai bientôt dans ma propre enquête ethnonaturaliste et dont je souhaiterais vous livrer mon expérience comme un ultime remerciement à cet enrichissement...

\section{Le dessin comme outil pour appréhender la diver- sité du monde vivant}

Son enseignement sur l'utilité multiple qu'il prêtait au dessin m'avait plus que séduit, il m'avait absolument convaincu. Cependant, je ne pouvais me mentir à moi-même! Même si, comme tout naturaliste en herbe, je nourrissais le rêve de dessiner les plantes et les animaux que j'allais découvrir en Papouasie, comme le faisait précisément Jacques Barrau ou encore Johann Walter ${ }^{1} \mathrm{au} \mathrm{XVII}^{\mathrm{e}}$ siècle, je devais me résigner à ma réalité : je ne disposais pas de leur talent. Et cette amère réalité avait de quoi m'inquiéter: n'allais-je pas à la rencontre d'une des forêts tropicales les plus luxuriantes de notre planète en abritant plus de 20000 espèces végétales ? Comment allais-je poursuivre ma réflexion ethnobotanique avec les Kasua, sans les fameux dessins? Ces questions me hantèrent, jusqu'au jour où je réalisais que la solution se trouvait là, devant mes yeux : dans le vieux Yashika qu'Haudricourt m'avait donné. Utilisant des films au format six/six, c'est-à-dire d'un grand format, il me suffisait de me procurer une boîte de développement et d'employer les négatifs comme substituts aux dessins. Cette " trouvaille » technique eut l'effet miraculeux escompté : elle effaça mes inquiétudes ethnographiques et avec, l'image déroutante d'une forêt tropicale immensurable.

Cependant ma sérénité, lâchement conquise, sera vite et durement éprouvée par la réalité floristique et écologique du territoire des Kasua ! Non seulement leurs terres couvraient une superficie de plus de $3000 \mathrm{~km}^{2}$, faisant de leur territoire tribal le plus grand de la région Bosavi, mais de surcroît, s'étendaient sur plus de $2000 \mathrm{~m}$ de dénivellation.

Autrement dit, et sans négliger les trois cents sous-canopées formées spontanément par les palmiers Metroxylon sp, les Kasua évoluaient dans trois écosystèmes forestiers distincts ! : la forêt des basses terres alluviales considérée par les botanistes comme la forêt la plus «luxuriante » de la Papouasie (Paijmans, 1975 : 10), où « la flore est extraordinairement riche, particulièrement en espèces d'arbres »(Clunie, 1978 : 5) ; la forêt tropicale des basses collines reconnaissable par sa canopée plus vaste et variée, et enfin, la forêt des basses montagnes caractérisée par une canopée moins haute mais très fermée du fait de la plus grande densité des spécimens. Autant dire que sur ces terres kasua, le règne végétal exalte dans la démesure, suscitant aussi un sentiment d'infiniment vert, car à sa différence, le règne humain se révèle dans la discrétion : le manteau de verdure recouvre plus de $99 \%$ de cette aire. L'océan végétal se voit donc à peine effleuré par les activités de subsistance kasua où l'horticulture itinérante occupe, il est vrai, une place bien subalterne comparée à celle de l'exploitation du palmier sagoutier, où les pratiques d'essartage exhibent volontairement une économie d'abattage des arbres dans la volonté affirmée de ne pas entraver le cycle de décomposition produit par l'extraordinaire humidité ambiante (Brunois, 2001 : 270-280). En effet, selon les Kasua, c'est en entretenant son taux à son plus haut niveau que l'humidité s'avère efficacement régénératrice en exerçant son pouvoir omnipotent sur tout ce qui est susceptible de se décomposer et de participer généreusement à la grande fabrique du précieux humus, nommé " graisse fertile » en kasua. La régénération et la préservation des centaines d'espèces d'arbres peuplant leur massif forestier reposait ainsi sur un simple secret : l'humidité 2 . Et ce n'est certainement pas étonnant si mon enquête ethnobotanique fut conditionnée par la même loi écologique gouvernant son objet, le règne végétal. En effet, il suffit de quelques semaines pour que ce facteur abiotique, aussi bienfaisant qu'impitoyable, s'en prenne fatalement au Yashika d'Haudricourt, me dérobant ainsi, et par erreur, mon seul et durable outil de réflexion, m'abandonnant à mes seules plantes herborisées que je n'osais même plus sortir de l'herbier placé au dessus du foyer, de peur qu'en interrompant leur enfumage, elles succombent à leur tour, à l'appel de la régénération forestière ! Certes, je pouvais consulter mes carnets de notes et me remémorer certains critères que j'avais pris soin de spécifier. Je pouvais également apprendre

1. Je cite Johann Walter particulièrement, car il est, à ma connaissance, le premier peintre naturaliste européen — il œuvrait au profit du comte Jean de Nassau Idstein - à reproduire, en 1623, un « casoar à casque qui avale tout rond ce qu'il dévore », soit un Casuarius casuarius!

2. Comme le fait Jacques Barrau (1965), les Kasua opposent l'humidité à la sécheresse, synonyme d'une mort irréversible au sens où le corps mort ne peut être recyclé. 
leur nom respectif. Mais cette mémoire restait cruellement vide de sens imagé. Un trouble que la nomenclature des plantes kasua ne faisait d'ailleurs qu'accentuer en présentant un polysémisme formidable : un arbre pouvant partager son nom avec une liane, avec un marsupial ou encore un humain. Pas davantage, ne pouvais-je me rassurer par l'observation in situ, c'est-à-dire en forêt. Car, si le règne végétal s'impose à vous, il vous dévoile moins volontiers les individus la composant. L'absence de recul, la densité de la canopée ne permettent pas à l'œil humain d'avoir une vision de l'être arborescent dans son entité. La diversité végétale se cherche dans la subtilité, et les Kasua allaient rapidement m'apprendre à observer cet invisible, c'est-à-dire, à combler ma frustration visuelle en faisant appel à mes autres sens, comme sentir qui équivaut souvent à voir dans l'enquête botanique : certaines essences se reconnaissant au seul parfum de leur écorce. Mais ce critère d'identification, aussi efficient soit-il, ne s'appliquait certainement pas aux centaines d'espèces distinctes déjà botanisées.

\section{L'identification aux plantes grâce au dessin}

Le constat était donc consternant. J'avais beau herboriser jour après jour, la forêt des Kasua me restait encore étrangère. Ma recherche stagnait au sens où ma propre capacité à appréhender et donc à ressentir les différentes espèces végétales stagnait. Et bien sûr, c'est cette terrible conclusion qui fit renaître le souvenir de mon entretien avec Jacques Barrau. Ses mots résonnaient dans mon esprit comme autant d'espoirs de progresser dans mon enquête botanique, de la régénérer peut-être. D'ailleurs, l'absence d'alternative me libéra instantanément de mes complexes graphiques, nés sans doute du caractère hybride que l'Occident prête à cet outil, et dont l'artistique était précisément insignifiant dans cette société qui laisse à la nature forestière et à ses esprits le privilège de l'invention créatrice. Néanmoins, mes premiers pas dans le dessin naturaliste furent fort laborieux. $\mathrm{Si}$, comme Degas l'affirmait, « le dessin n'est pas la forme, il est la manière de voir la forme », je voulais que cette représentation de la plante, de sa feuille ou de ses fruits, soit la plus objective possible. Or, ne possédant qu'un seul cahier à dessin, je ne pouvais me permettre de reproduire les feuilles dans leur format réel, certaines mesurant plus de 70 $\mathrm{cm}$ ! Le premier exercice consista donc à apprivoiser l'espace à dessiner et mon regard sur la plante, laissant à ma main le rôle de pantogra- phe. C'est ainsi que, croquis après dizaines de croquis, je réalisais qu'une certaine harmonie s'était instaurée entre les plantes, mon œil et ma main, qu'elle devenait même spontanée. En fait, je me surprenais à réaliser la confidence de Jacques Barrau, selon laquelle la reproduction manuelle permettait de rendre l'être ainsi dessiné - quelle que soit sa dimension réelle - , proportionnel à soi, à sa dimension, conférant au dessinateur naturaliste ce sentiment « d'être plus en harmonie ». En dessinant, mon œil regardait enfin l'arbre dans son entier. Puis une étrange association s'établit incidemment, celle entre «l'œil objectif » et l'identification subjective à l'être que je dessinais. Au fil des heures passées solitairement avec les feuilles ou les fruits, les essences végétales me devenaient familières comme des amies participant à mon univers intime, à mon être "imprégné » désormais de leur présence, de leur existence, de leur identité singulière. Le dessin botanique a cela de généreux qu'il vous apprend à ressentir combien la flore a le sens de la création, du détail, légitimant les centaines de taxons reconnus par les Kasua et dont chacun des génériques faisait enfin sens à mes yeux. Décidément, Jacques Barrau ne se trompait pas lorsqu'il avançait que le dessin permet d'être plus attentif à ce que l'on étudie ! Une impression partagée par de nombreux artistes s'étant adonné au dessin des plantes. Le peintre Kelly, par exemple, ne reconnaissait-il pas que ce dessin lui permit « d'être au plus près de la perception »? Comment en douter ? D'autant que le dessin botanique recèlera des implications épistémologiques encore plus remarquables pour l'ethnologue que je devenais.

En effet, l'étrange identification aux arbres, qu'instaurèrent subtilement leurs reproductions graphiques, me rapprocha étonnamment de la perception kasua qui voit dans les centaines de plantes autant d'individualités rivalisant d'originalité et donc de personnalité, comme en témoigne d'ailleurs leur systémique non inclusive. Le mythe de leurs origines ne les assimile-t-il pas à des danseurs mi-humain mi-arbre, dont les décorations corporelles et personnalisées se confondent aujourd'hui aux caractères physionomiques particuliers qu'exhibe chacune des espèces arborescentes?

\section{Le dessin comme outil pour appréhender la place de l'Homme dans le monde vivant}

L'épaisseur de l'herbier et de mon cahier de dessin mesurait le savoir botanique des Kasua. Ils ne contenaient pas cent, deux cents ou encore 
trois cents plantes nommées, croquées distinctement, mais plus de 500 essences végétales, pour chacune desquelles les Kasua attribuaient un, voire plusieurs usages puisque, pour l'ensemble de cette flore herborisée, je totaliserais finalement 1410 emplois distincts (Brunois, 2001 : 295 ; Bahuchet, $2000: 135$ ). Cette formidable utilité, prêtée au règne végétal, légitimerait certainement aux yeux de Jacques Barrau l'assimilation de la société kasua à « une civilisation du végétal » (Barrau, 1967 : 279 citant Gourou, 1965-1966 : 311), témoignant d'un temps, toujours actuel, de « l'âge du bois, du bambou, des fibres » (Barrau, 1967 : 279 citant Sauer, 1947 : 6 ; Corner 1960 : 39). De fait, pour ces individus, la forêt est par excellence "la Forêt-Matière » (Bahuchet, 2000 : 135), où ils puisent, quotidiennement, tous leurs moyens d'existence ${ }^{3}$, conférant à leur culture matérielle ce caractère étonnamment éphémère (Bahuchet, 1990), imposant à ma recherche ethnobotanique une nouvelle exigence : comprendre les logiques gouvernant ces innombrables relations au monde végétal. Car, logiques il y a! Elles s'expriment dans les différents choix sélectifs qu'exercent les Kasua en fonction des usages recherchés et des caractéristiques qu'offrent les plantes ou partie de ces plantes, tels le tronc, l'écorce, l'aubier, les exsudations, le fruit, ou encore les feuilles. Elles sont nécessairement fort variées compte tenu, précisément, de l'immense diversité des emplois que font les Kasua du règne végétal, mais aussi, de l'extraordinaire diversité floristique que présente ce même règne végétal. Un exemple révélateur nous est offert par les matériaux utiles à la construction de leur fameuse grande demeure mesurant $40 \mathrm{~m}$ de long sur $20 \mathrm{~m}$ de large et s'élevant à plus de $15 \mathrm{~m}$ de hauteur! La quantité d'arbres nécessaires à son aménagement est impressionnante : le chiffre côtoie les 760 ! Et pourtant, seules 200 espèces sont considérées comme utiles à son édifice, et ce n'est qu'une première sélection. Parmi ce stock limité d'arbres, une soussélection est automatiquement et rigoureusement établie selon la destinée du tronc. Devenir l'un des cent poteaux porteurs de la gwane sosolo ou "grande maison », n'est pas à la portée de tous ! Une dizaine d'entre eux uniquement pourront prétendre à cette noble fonction d' « arbres de fer ${ }^{4}$. Pas davantage, prêter son écorce aux parois murales ou à la couverture du plancher n'appartient à n'importe quelle espèce pourvue d'écorce. Tout au contraire ! Cette fonction exige de l'arbre d'arborer des caractéristiques précises que seules deux espèces remplissent pleinement. D'ailleurs, leur nomination respective révèle combien la logique sélective a ici atteint son idéal : le générique de la plante se confondant à celui du matériel de construction : paroi murale, plancher. Leur cas n'est pas isolé, bien sûr ! La nomenclature de la flore recèle de nombreux autres exemples où le nom de la plante se confond à l'objet, au remède ou encore au rituel tiré de son exploitation préférentielle, empêchant ainsi d'affirmer avec certitude une prévalence de l'humain sur le végétal ou inversement du végétal sur l'humain ; illustrant, s'il le fallait, combien ces logiques ne procèdent pas d'une relation unilatérale de l'homme sur le végétal, mais bien d'une expérience relationnelle interactive entre l'être humain et l'être végétal, autrement dit, d'une relation où la plante, au même titre que l'individu kasua, agit sur l'autre, forte de sa personnalité. Comprendre les logiques relationnelles qu'entretenaient les Kasua avec chacune des centaines d'espèces herborisées exigeait donc de comprendre non pas « quoi » était la plante utilisée, mais bien « qui » elle était. En somme, cela signifiait, méthodologiquement parlant, que pour saisir chaque usage inventorié ou observé, il me fallait avoir la plante devant mes yeux. Une exigence insurmontable dans l'enquête de terrain au cours de laquelle le recueil des informations n'est jamais synchronique. À moins, bien sûr, de disposer de l'outil graphique qui « prolonge la réflexion ». En effet, cette propriété, que prêtait Jacques Barrau au dessin, est inestimable dans l'étude des relations réciproques - et non unilatérales - liant l'homme et le végétal ${ }^{5}$ car, en prolongeant la mémoire du sujet dessiné comme la mémoire des émotions suscitées par sa reproduction graphique, cet outil permet de tenir compte, précisément, de la personnalité de la plante dans la compréhen-

3. L'exemple kasua confirme la critique énoncée par Dwyer et Minnegal (1991) à l'encontre de l'opinion selon laquelle une société de chasseurs-cueilleurs ne pourrait vivre en milieu tropical humide sans la pratique horticole (Headland 1987 ; Bailey et al. 1989). En effet, d'après ces deux auteurs, et à l'appui de leur ethnographie menée auprès de la société Kubo (PNG), cette opinion néglige l'exploitation du palmier sagoutier qui permet aux sociétés des Basses Terres de Papouasie Nouvelle-Guinée de développer précisément un modèle de subsistance non agricole, et de développer leurs connaissances du milieu végétal «sans être constamment menacés de famine » (Barrau $1967: 289$ ).

4. D'ailleurs, l'incendie qui allait consumer en 1996 la dernière grande demeure traditionnelle donnera raison à la sélection faite par ses bâtisseurs : seuls les poteaux porteurs résistèrent aux flammes ravageuses. Seul aussi, l'immense dessin, qu'en avait réalisé mon compagnon, résista à mon oubli de compter le nombre de troncs utiles à sa reconstruction. Cette expérience, qui n'est pas à mon honneur, témoigne, paradigmatiquement, du rôle mnésique qu'accordait J. Barrau à ses dessins.

5. Précisément l'objet d'étude de l'ethnobotanique (Barrau 1967 ; 1971). 
sion des usages que lui attribuent les humains. En d'autres termes, l'outil graphique permet d'appréhender, de façon sensible, les logiques gouvernant l'interaction entre les deux règnes.

\section{L'apport du dessin dans les logiques interactives liant l'Homme au végétal}

Nombreuses de ces logiques kasua reposent effectivement sur des qualités sensorielles particulières qu'exhibent les plantes ou certaines de leur partie, et qui ne peuvent échapper au dessinateur "attentif à ce qu'il étudie », attentif au moindre détail, aussi subtile soit-il. Cette quête de la particularité est d'ailleurs constante. Non pas seulement parce qu'elle permet au dessinateur de distinguer telle essence d'une autre ; mais aussi, parce qu'elle seule le tient en éveil. La reproduction graphique de centaines de plantes serait terriblement monotone si elle n'était rythmée régulièrement par l'étonnement que suscite l'observation d'une qualité floristique originale. Ces émotions inattendues participent même, et pleinement, à l'enquête ethnobotanique car, en se reproduisant, elles instituent des réflexes de questionnement pour lesquels les réponses deviennent souvent prévisibles, révélant, par là même, combien la particularité "émouvante" de la plante relève bien d'une logique relationnelle. J'en donnerai quelques exemples. La première est certainement la plus enivrante. Il s'agit bien sûr de la logique des odeurs que dégagent certaines plantes, leur bois, leur écorce, ou encore leurs feuilles. Ces espèces " odorantes» sont rares, mais le dessinateur, qui se voit soudainement embaumé par le parfum du sujet dessiné, ne peut manquer de les reconnaître. Pas davantage n'ont-elles laissé les Kasua indifférents. Toutes ces plantes en effet offrent des usages gouvernés par la senteur qu'elles exhalent. Cependant, ces usages varient selon la qualité même de leur parfum, jamais considéré comme malodorant ${ }^{6}$. Les plantes aux senteurs les plus sucrées serviront d'arômes pour la cuisson des aliments ou pour l'eau de source contenue dans les entrenœuds de bambou. Celles aux senteurs épicées seront utilisées pour soigner une toux, un enrouement, pour éclaircir la voix d'un danseur et l'olfaction d'un chien, ou encore, pour faire fuir simplement les poux. En revanche, les plantes dégageant un parfum très puissant reçoivent un traitement particulier, précisément en raison de cette particularité. Considérées comme appartenant aux esprits, ces essences sont employées exclusivement dans les contextes ritualisés où les esprits prennent place, comme la chasse, la guerre, les attaques de sorcellerie, les initiations... Ainsi, en retenant le parfum comme trait de personnalité de la plante, la logique des odeurs voit sa portée limitée en excluant toutes les autres essences inodores. Celles-ci participent à d'autres logiques comme celles des couleurs que revêtent le tronc, l'exsudation, la feuille ou encore le fruit. La couleur peut susciter une émotion similaire à celle d'une odeur, au sens où sa vision peut surprendre. Ainsi en est-il, par exemple, quand une feuille rougeâtre se présente au dessinateur, trahissant une des exceptionnelles essences caduques de la forêt, ou encore une inflorescence aux pétales jaunes, une sève d'un jaune ambré ou d'un rouge écarlate, un fruit à l'épicarpe rouge étincelant, un spadice d'un blanc immaculé... La palette des couleurs n'est pas si dense, mais chacune d'entre elles provoque, chez le dessinateur, la même réaction émotive. Elle se légitime bien évidemment au regard de l'environnement végétal et des teintes qu'il arbore. Assimiler la forêt tropicale à un océan infiniment vert ne relève pas de la seule métaphore! C'est négliger seulement que sous la canopée règne l'infiniment brun. Et, peut-être, est-ce par cet excès de référent que les Kasua n'ont pas de termes pour désigner ces deux coloris. En fait, si l'on excepte le noir également nommé, seuls le blanc, le rouge et le jaune - les couleurs dont se recouvre plus volontiers le monde végétal comme les substances humaines - sont reconnus comme des couleurs vives et, plus fondamentalement, comme des couleurs " vivaces ", au sens où elles agissent sur l'individu qui les voit, les touche, les consomme. Et c'est bien en tant que telles qu'elles participent à une logique relationnelle gouvernant les usages des plantes dont une partie ou plusieurs de leurs parties sont teintées de ces nuances. Ainsi, tout ce que le végétal a de rouge ou de blanc sera utilisé ou au contraire interdit — dans tous les cas, soumis à des rituels -, dans les contextes individuels ou collectifs liés à la condition de l'humain réel, c'est-à-dire, à sa condition marquée par la reproduction sexuelle et la mort. En revanche, tout ce qui dans la flore revêt la couleur jaune sera employé dans les contextes où la condition invisible de l'être humain doté d'un double cosmologique, et du cosmos forestier en général, sera en jeu.

6. À l'exception d'une seule essence qui, inodore vivante, dégage une odeur nauséabonde quand son bois coupé, c'est-à-dire mort, est consumé par le feu. Cette propriété particulière explique que cette essence soit exclue des 53 espèces utilisées comme bois de chauffe ou encore de celles utiles à la construction des maisons. 
Cette logique des couleurs concerne plus d'une centaine d'espèces végétales, aussi, je retiendrais deux exemples dont les dessins m'ont sensibilisée à cette logique. En effet, comment aurais-je pu réaliser la portée de la logique du rouge et du blanc, si plusieurs mois avant que les Kasua ne me «narrent» l'usage de l'arbre sekene dans le rituel initiatique, consacrant l'accès des frères et des sœurs à la reproduction sexuelle, je n'avais croqué, avec surprise, son fruit rouge vermillon, formant à maturation un vagin denté, et son tronc étonnamment blanc? Le second cas coloré est, je crois, aussi éloquent : il concerne l'arbre monumuna, terme désignant remède, médicament. Comme son nom l'indique, cette essence est la plante médicinale par excellence! Elle soigne toutes les maladies, sachant que pour les Kasua, les pathologies que subissent les individus réels sont toujours dues à leur double cosmologique ou aux actions des esprits occupant le monde invisible. Rencontrée en forêt, cette essence prodigieuse passerait inaperçue : la robe de son tronc est brune, son feuillage vert. Et pourtant, son inflorescence est bien surprenante: généreuse car formée en hampe, elle revêt les couleurs d'un soleil éclatant. Aussi, n'est-ce pas un hasard si seuls ses organes foliacés sont dotés du pouvoir curatif. Frottés énergiquement sur la peau du patient, au point de provoquer des sensations de brûlures, ils sont censés répandre leurs bienfaits de manière homogène dans tout son corps, prenant exemple, peut-être, sur les graines du fruit qui, contenues dans le corps étroit du follicule, vont germer simultanément ( $c f$. Figs. 3, 4 et 5).

"L'invention extraordinaire de la nature " (Haudricourt, com. pers.) qui semble, ici, s'être exprimée comme un peintre impressionniste, en apposant sur la toile végétale des tâches de couleurs éparses, a bien sensibilisé les Kasua. C'est avec une immense fierté, d'ailleurs, que les danseurs et danseuses recouvrent leur corps de ces mêmes couleurs, de ces mêmes décorations floristiques dont s'étaient revêtus les ancêtres arborescents. Leur costume est un véritable hymne au végétal dont certaines essences le composant, comme entre autres deux cordylines particulières, ont été choisies pour la structure originale qu'elles déploient. La structure du végétal ${ }^{7}$ participe en effet d'une autre logique relationnelle. Elle gouverne sans aucun doute le plus grand nombre d'usages que tirent les Kasua de la flore. Il y a bien sûr la structure que le bois vivant ou mort oppose à la main du coupeur, du bâtisseur, du navigateur ou du confectionneur d'armes. Il y a bien sûr la structure de l'écorce ou plus précisément, la structure des écorces avec rhytidome. Celles convoitées par les femmes pour la confection des filets de portage ou des sacs à sagou et qui présentent un rhytidome se détachant en fines lanières seront précisément évitées par les hommes en quête d'une structure plus épaisse et crevassée longitudinalement pour couvrir le plancher ou élever les parois de la demeure. Il y a enfin la structure de la feuille et du fruit dont la logique me fut également révélée par le dessin. Par exemple, la surprenante douceur veloutée d'une feuille dont le limbe est recouvert de poils sera automatiquement prisée par une jeune maman pour la toilette de son nourrisson ; celles dont le limbe offre un aspect lisse ou caoutchouteux se transformeront en l'espace de quelques minutes en tasse d'appoint ou encore en toile pour empaqueter les aliments, fonction que remplissent également les longues feuilles des monocotylédones aux nervures si particulièrement longitudinales et non ramifiées. La structure sèche ou charnue du fruit induira des usages tout aussi précis. Les gousses, follicules ou encore capsules non fendues et qui, agitées, produisent un surprenant son tambourinant, seront systématiquement recherchées à l'annonce d'une cérémonie, leurs graines servant à la confection des hochets. Les fruits présentant un épicarpe excessivement dur - structure éprouvée quand il me fallait le casser avec le dos de ma hache pour découvrir et dessiner ce qu'il renfermait - serviront de leurre naturel à la chasse au casoar, seul animal, avec l'homme, apte à les consommer et à participer ainsi à la diffusion de ces essences singulières au sein de la forêt. Ce dernier exemple me conduit finalement à la dernière logique gouvernant les usages que je mentionnerais. Elle procède du même principe interactif, seule l'identité de l'être qui rentre en interaction avec la plante change.

\section{Le dessin comme révélateur d'une autre dimension relationnelle: celle liant la faune, la flore et les Hommes.}

L'utilité des plantes chez les Kasua ne peut se comprendre, en effet, que dans un sens plus large que celui traditionnellement entendu, c'est-àdire, « humanocentrique ». Pour les Kasua, une plante est aussi utile car utile aux animaux cohabitant dans leur massif forestier. J'ai relevé ainsi 483 usages que tire la faune de la flore herborisée (Brunois, 2001 : 133). Ils sont d'ailleurs aussi 
variés que ceux de l'humanité et offrent à certaines plantes une polyvalence fonctionnelle tout aussi surprenante. Il y a, bien sûr, les plantes alimentaires dont la faune, à plus de $80 \%$ phytophage, est friande. Toutefois, chaque animal manifeste des préférences comme le suggèrent, dans mes dessins, les traces des coups de bec sur les fruits complexes, les galeries creusées dans les tiges ramifiées de certaines plantes comme l'hoposa, les feuilles dont le limbe a été grignoté ou encore mâché pour en absorber la sève. Mais il y a aussi les plantes utiles à la construction comme la liane banabe meï qu'utilise de manière préférentielle l'oiseau jardinier pour aménager son nid à allée, ou encore les innombrables brindilles que sélectionnent et ramassent patiemment les mégapodes pour bâtir leur immense nid d'incubation. Le goût du confort conduit souvent les truies sauvages à cueillir les frondes des quelques fougères odorantes pour aménager leur litière, tandis que le couscous tacheté voit dans la liane peyo wale meï son moyen de transport idéal, et dans les lianes épineuses, des grattoirs efficaces. La liste est bien trop longue pour la restituer fidèlement et révéler ainsi combien " en vivant au contact étroit du monde végétal », les Kasua sont «devenus de remarquables naturalistes auxquels peu d'aspect de la vie végétale a échappé » (Barrau, 1967 : 278). Ce qui importe ici de noter, c'est que nombreuses plantes utilisées par les animaux à des fins particulières dans les exemples cités : les lianes épineuses, les fougères odorantes, la liane banabe meï - reçoivent un traitement similaire des humains. Cette remarquable similitude suggérerait que les Kasua aient appris l'utilité qu'ils prêtent aux plantes, non seulement en étant attentif à leurs personnalités singulières, mais aussi en observant les usages qu'en tirent les animaux, c'est-àdire en observant les caractères personnels des plantes retenus par le monde animal. D'ailleurs, les Kasua ne s'en cachent même pas lorsqu'ils reconnaissent avoir tout bonnement copié sur le couscous ou l'oiseau jardinier. Est-ce vraiment un hasard, si les fruits qu'ils collectent sont précisément les mêmes que ceux consommés par le casoar, dont leur tribu porte le nom, kasua.

\section{Du dessin au dessein des plantes sauvages}

À la lecture de cet éloge du dessin naturaliste comme outil ethnographique, on réalisera l'omniprésence du regard de Jacques Barrau durant les trois années passées auprès des Kasua et des centaines de plantes animant leur magnifique massif forestier. Son regard, à l'image des couleurs végétales et de ses tableaux, était non seulement vivant mais aussi vivace : il agissait sur ma main. Lorsqu'un de mes dessins me procurait une douce satisfaction, je me surprenais même à anticiper notre future rencontre. Je m'imaginais dans son fameux bureau, lui soumettant avec une certaine fierté mes « œuvres » graphiques!, lui racontant avec un certain empressement le destin tragique du Yashika, ma terrible consternation devant mes premiers dessins, l'extraordinaire exaltation que le dessin me suscitait lorsqu'il me sensibilisait à une logique liant l'homme au végétal, ma conviction que pour comprendre le passage de «l'Homme cueilleur à l'Homme cultivateur », il faille considérer également les relations qu'entretient la faune, côtoyant l'Homme, avec le monde végétal. Oui, je m'imaginais lui révéler dans un enthousiasme désordonné tout ce que je vous ai révélé aujourd'hui, comme un magnifique remerciement pour m'avoir fait partager son amour du dessin naturaliste. Mais notre future rencontre, si souvent imaginée de la forêt, n'a jamais eu lieu. Et Jacques Barrau n'a jamais su que je ne recevrais jamais les identifications scientifiques du botaniste papou à qui j'avais pourtant confié deux exemplaires de mon herbier tant choyés, et que ce sont leurs dessins qui me dévoileront leur identité scientifique, permettant peut-être à ces plantes sauvages ainsi « croquées » d'être préservées des dents bien plus cannibales des scies industrielles (Brunois, 1999). Oui, heureusement, un petit dessin en dit parfois plus long qu'un discours... N'est-ce pas ce que nous évoque le dessin des enfants kasua ( $c f$. Figs. 6 et 7) ? Des Jacques Barrau en herbe, sans nul doute...

\section{REMERCIEMENTS}

Cet article repose sur un terrain mené dans la Province du Sud de la Papouasie-Nouvelle-Guinée, de Septembre 1994 à Mai 1997. Ma recherche fut rendue possible grâce à la contribution financière de diverses institutions que je tiens à remercier : le Ministère de l'Éducation et de la Recherche, la Mairie de Paris, la Fondation Yves Rocher, et enfin, l'Avenir des Peuples des Forêts Tropicales (Programme de la DG VIII. Commission Européenne).

\section{RÉFÉRENCES}

AdAm Hans Christian, 1999. Karl Blossfeldt. 18651932. Cologne : Taschen. 
BAHuchet Serge, 1990. Chez les Pygmées d'Afrique centrale, des outils de l'éphémère..., Corps écrit 35, pp. 13-20.

—, 2000. La Forêt-Matière, in S. Bahuchet (ed), Les Peuples des Forêts Tropicales Aujourd'hui. Une Approche Thématique, vol. 2, Bruxelles : APFT-ULB, pp. 135-157.

BARRAU Jacques, 1962. Les plantes alimentaires de l'Océanie. Origines, distribution et usages. Marseille, Annales du Musée colonial de Marseille, $7^{\mathrm{e}}$ série, vol. III à IX (1955-1961), 275 p.

_, 1965. L'humide et le sec. An essay on Ethnobiological Adaptation to Contrastive Environments in the Indo-Pacific Area, Journal of the Polynesian Society 74, pp. 329-346.

—, 1967. De l'homme cueilleur à l'homme cultivateur : l'exemple océanien, Cahiers d'Histoire Mondiale, Vol 10, n², Neuchatel, pp. 275-292.

_, 1971. L'ethnobotanique au carrefour des sciences naturelles et des sciences humaines, Bulletin de la Société Botanique Française 118, pp. 237-248.

BRUNOIS Florence, 1999. The ecological impact of the Wawoi Guavi Company's logging exploitation on the territory and society of the Kasua people. Vol. 4. Working Papers. Bruxelles, APFT-ULB.

_, 2001. Le Jardin du Casoar, la Forêt des Kasua. Influences des relations au milieu forestier tropical sur l'identité et les savoir et savoir-faire écologiques de la société Kasua. Grand Plateau de Papouasie Nouvelle Guinée. Thèse de doctorat. Paris : EHESS, $427 \mathrm{p}$.

Clunie N.M.U, 1978. The Vegetation in J. Womersley (ed), Handbooks of the Flora of Papua New Guinea, vol I, Melbourne University Press, Australie, pp. $1-11$.

Corner E.J.H., 1960. "Botany and Prehistory », Symposium of the Impact of Man on the Humid Tropic Vegetation, Papuasie Nouvelle-Guinée, Port Moresby, pp. 38-41.

Dwyer P. and Minnegal M., 1991. Hunting in Lowland, Tropical Rain Forest : Toward a Model of Non-agricultural Subsistence, Human Ecology, Vol 19, n², pp. 187-212.

Gourou P., 1965-1966. «Étude du monde tropical (géographie physique et humaine) », résumé des cours de 1964-1965, Annuaire du Collège de France, pp. 307-318.

Paijmans K., 1975. Explanatory notes to the vegetation map of Papua New Guinea, CsIro, Australie, Land Research series, $n^{\circ} 35$.

Sauer C.O., 1947. Early Relations of Man to Plants, The Geographical Review 37, I, pp. 1-25. 


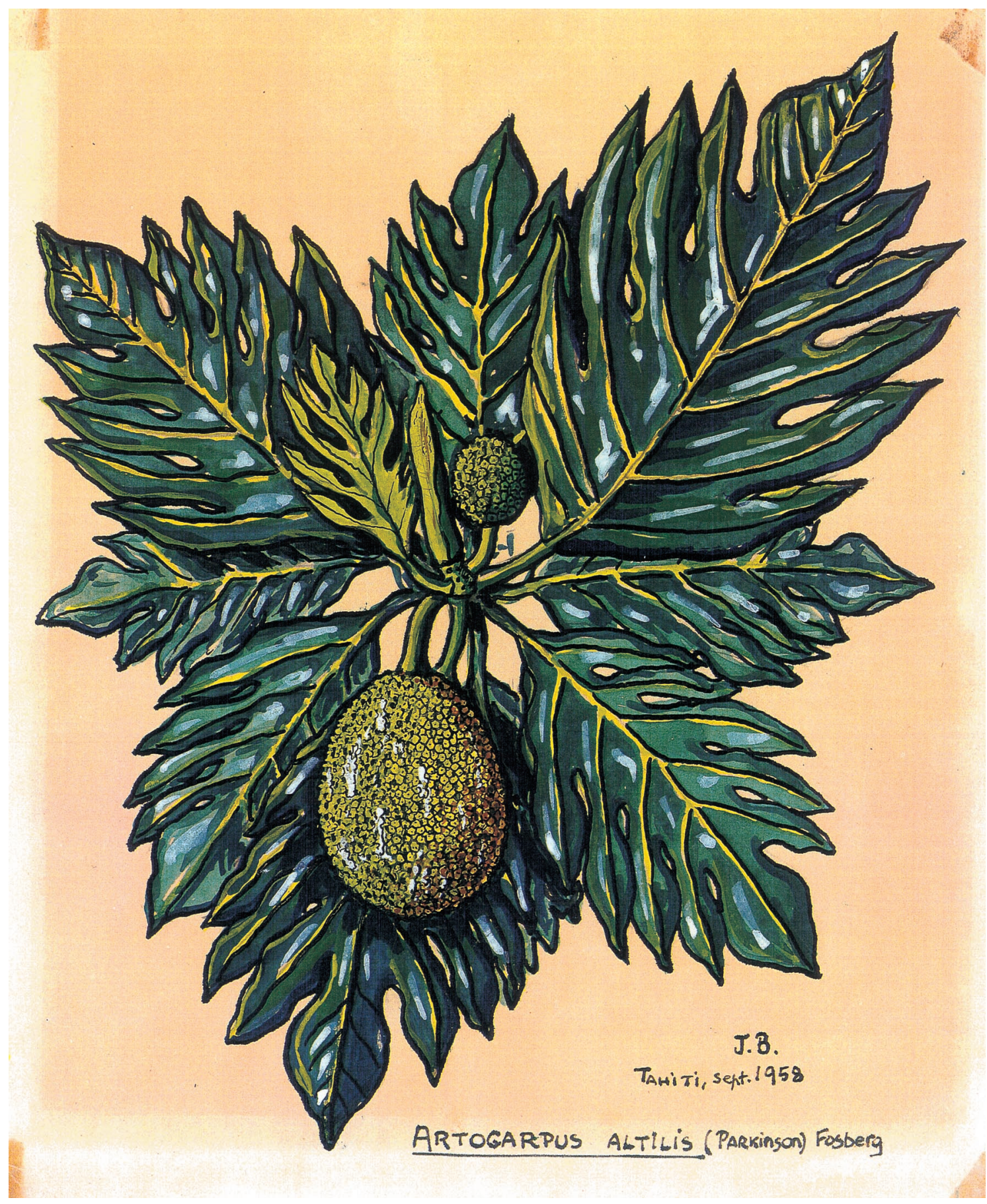

FIG. 1 - Gouache de Jacques Barrau (prêt d'Alice Peeters). 


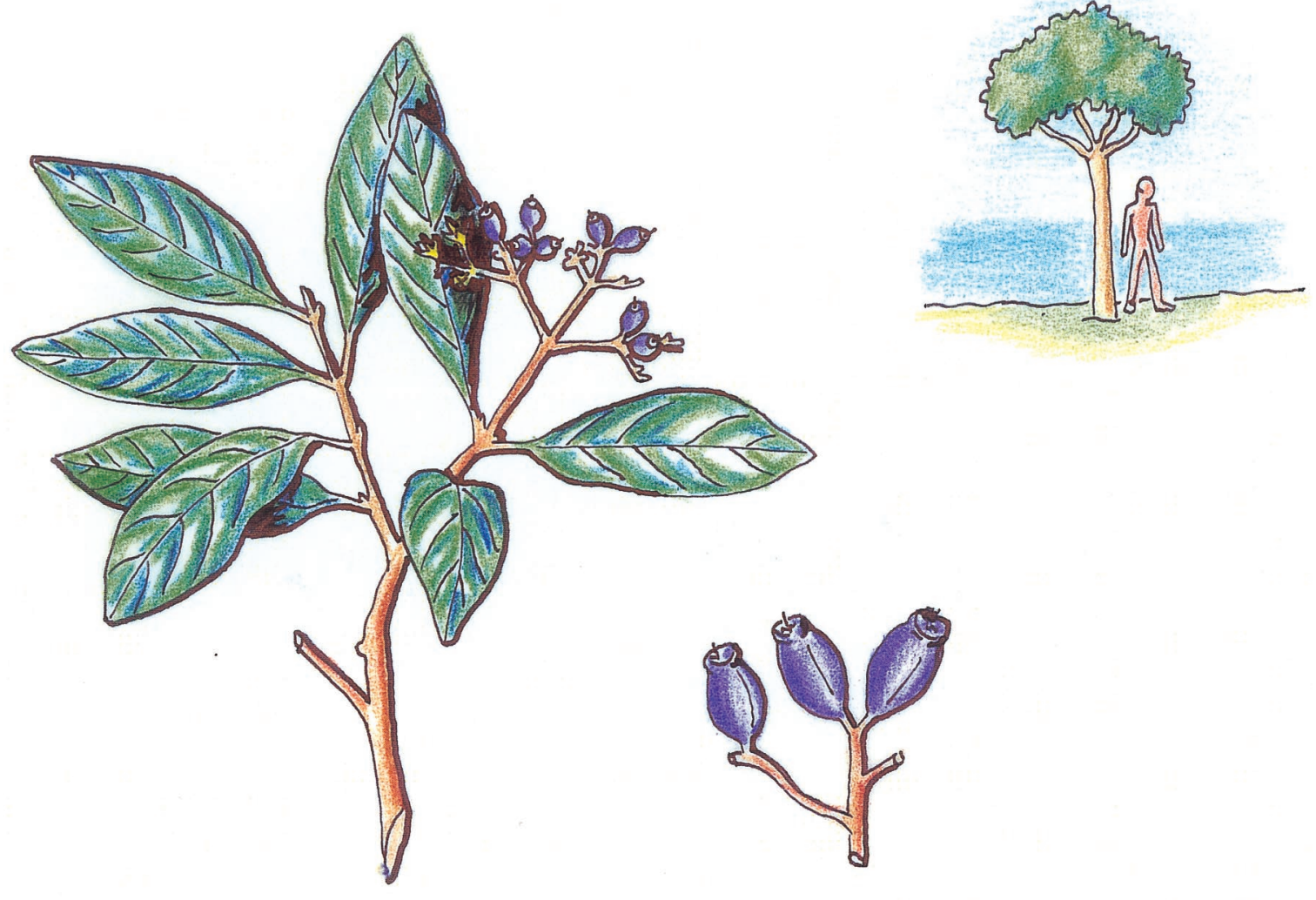

santal de la Nourelle Caledonie

Santalum acstro-caledonicum, Vieill.

Fig. 2 - Dessin de Jacques Barrau rehaussé au crayon de couleur (prêt d'Alice Peeters). 


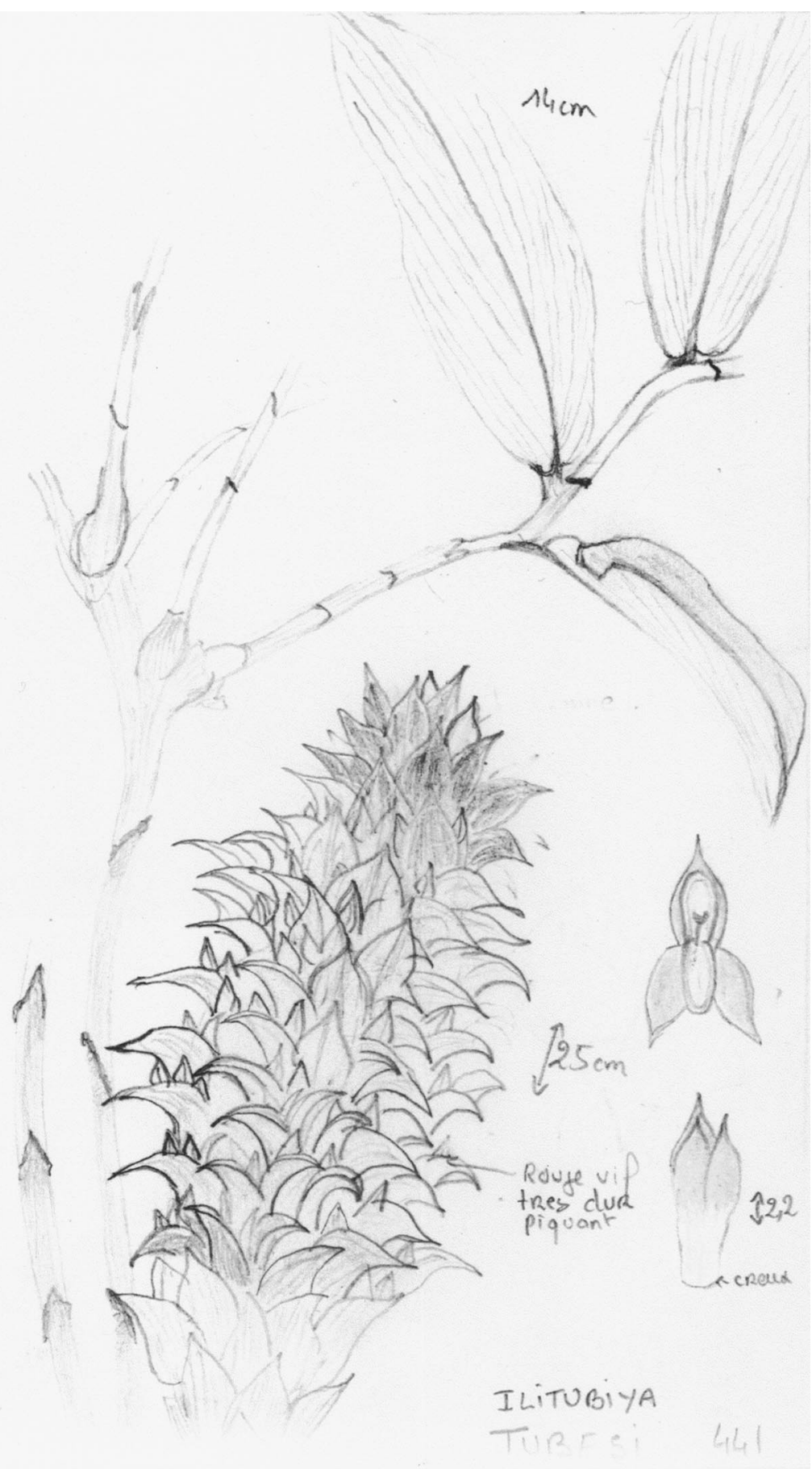

FIG. 3 - Éloge de la sculpture végétale. Dessin à la mine de plomb d'un rameau et d'une fleur d'ilitubiya par Florence Brunois. 


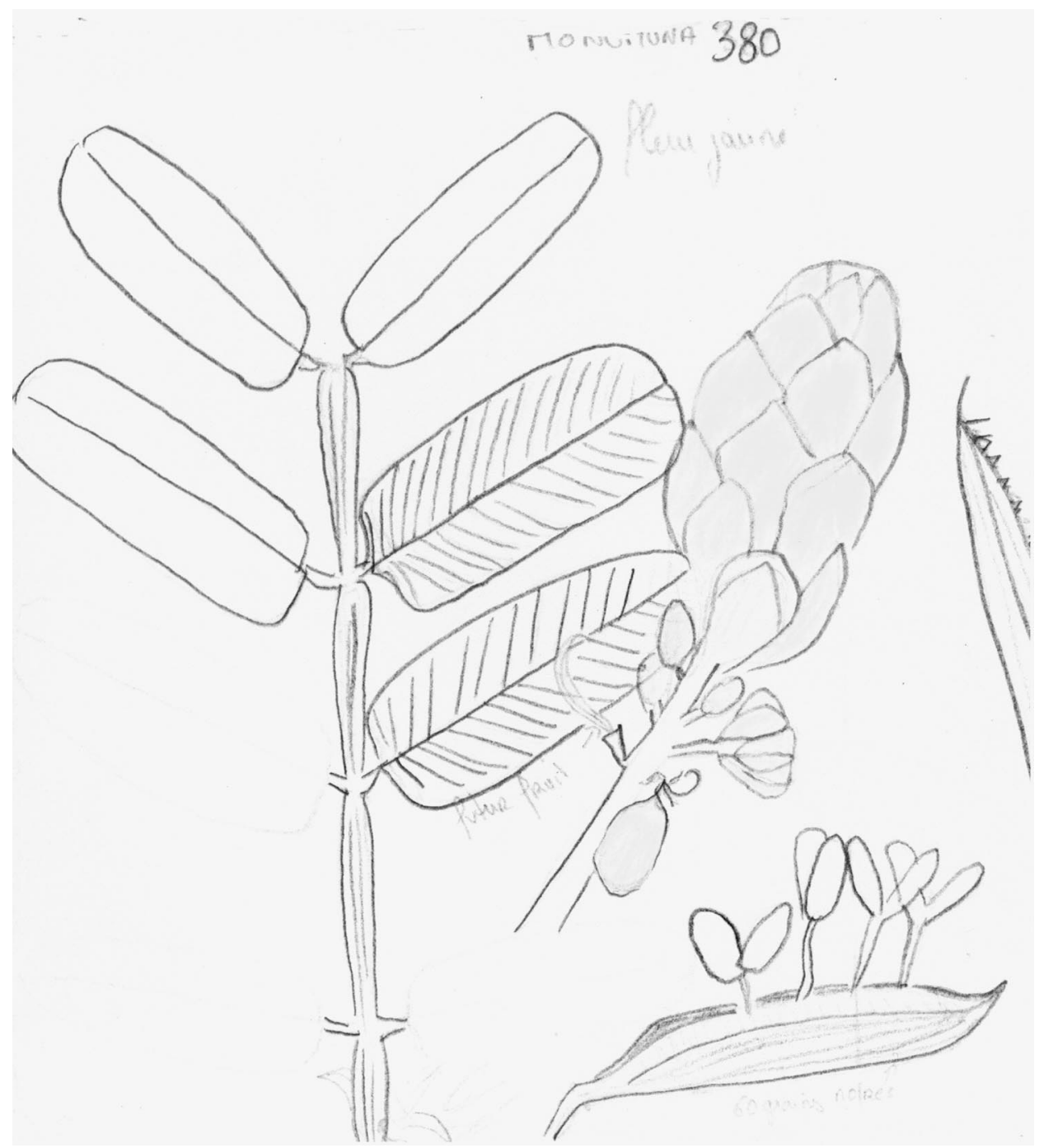

FIg. 4 - Éloge de la couleur végétale. Dessin à la mine de plomb d'une feuille et d'une fleur de monumuna par Florence Brunois. 


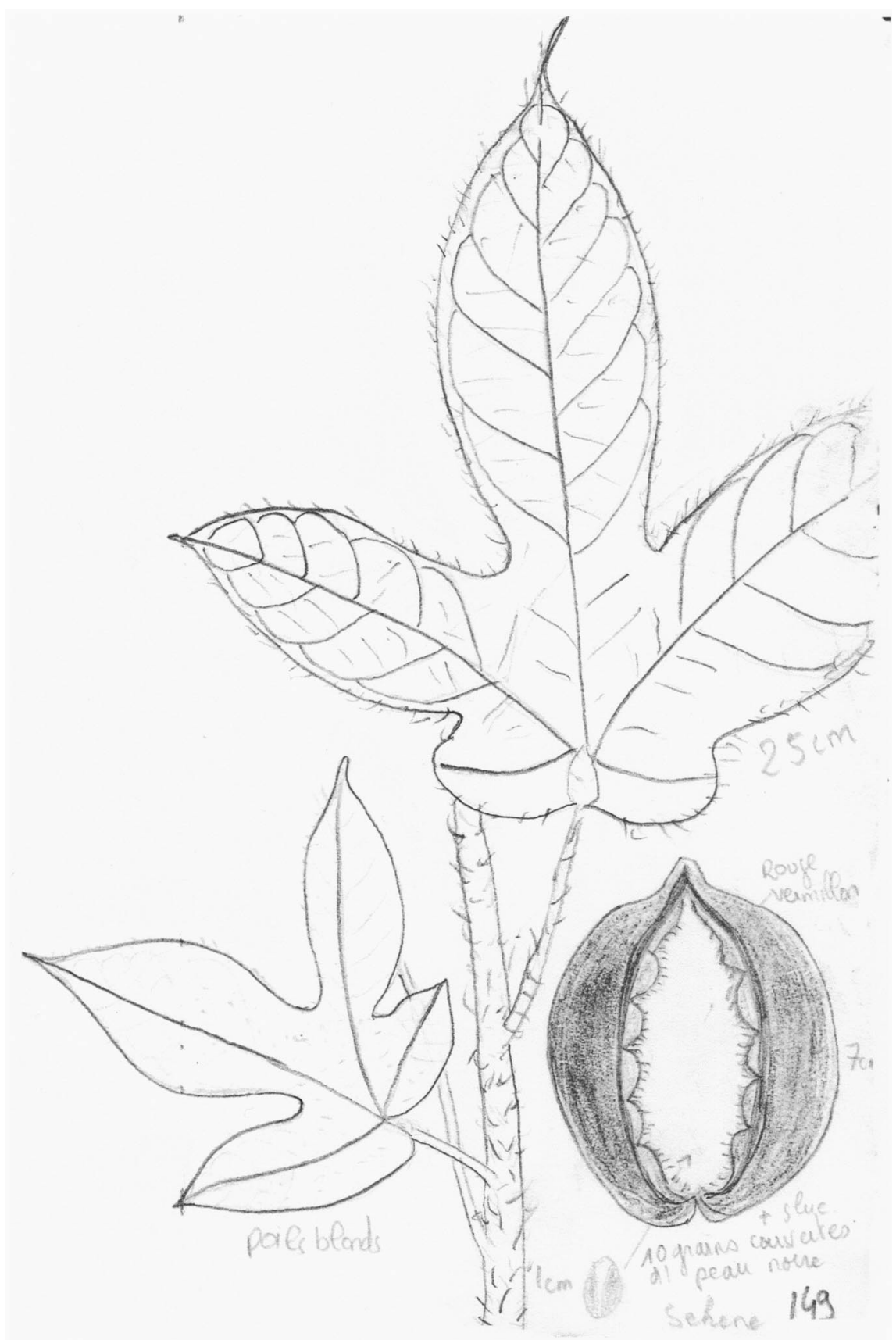

FIG. 5 -Éloge de la sculpture végétale. Dessin à la mine de plomb d'un rameau et d'une graine de sekene par Florence Brunois. 


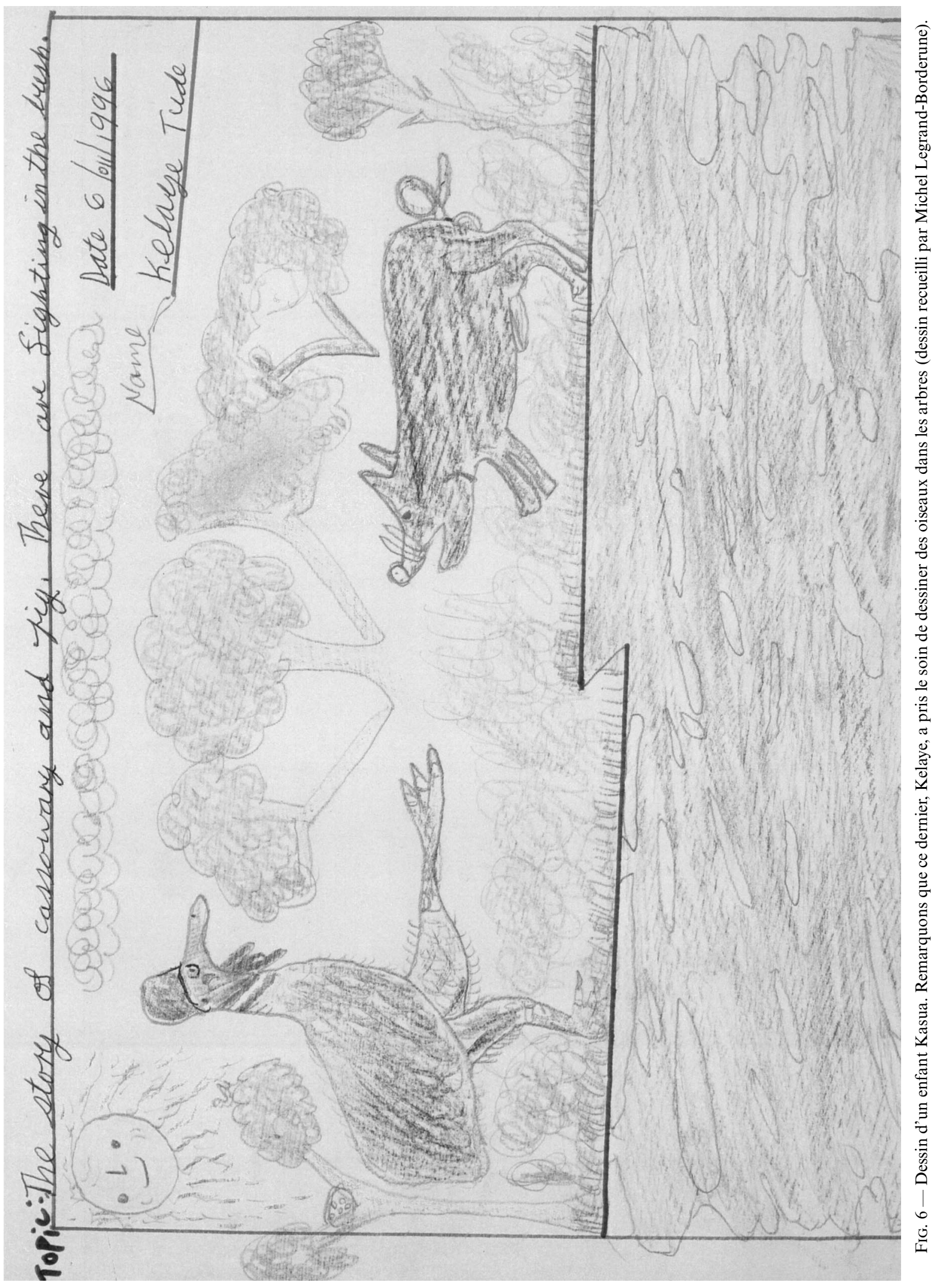




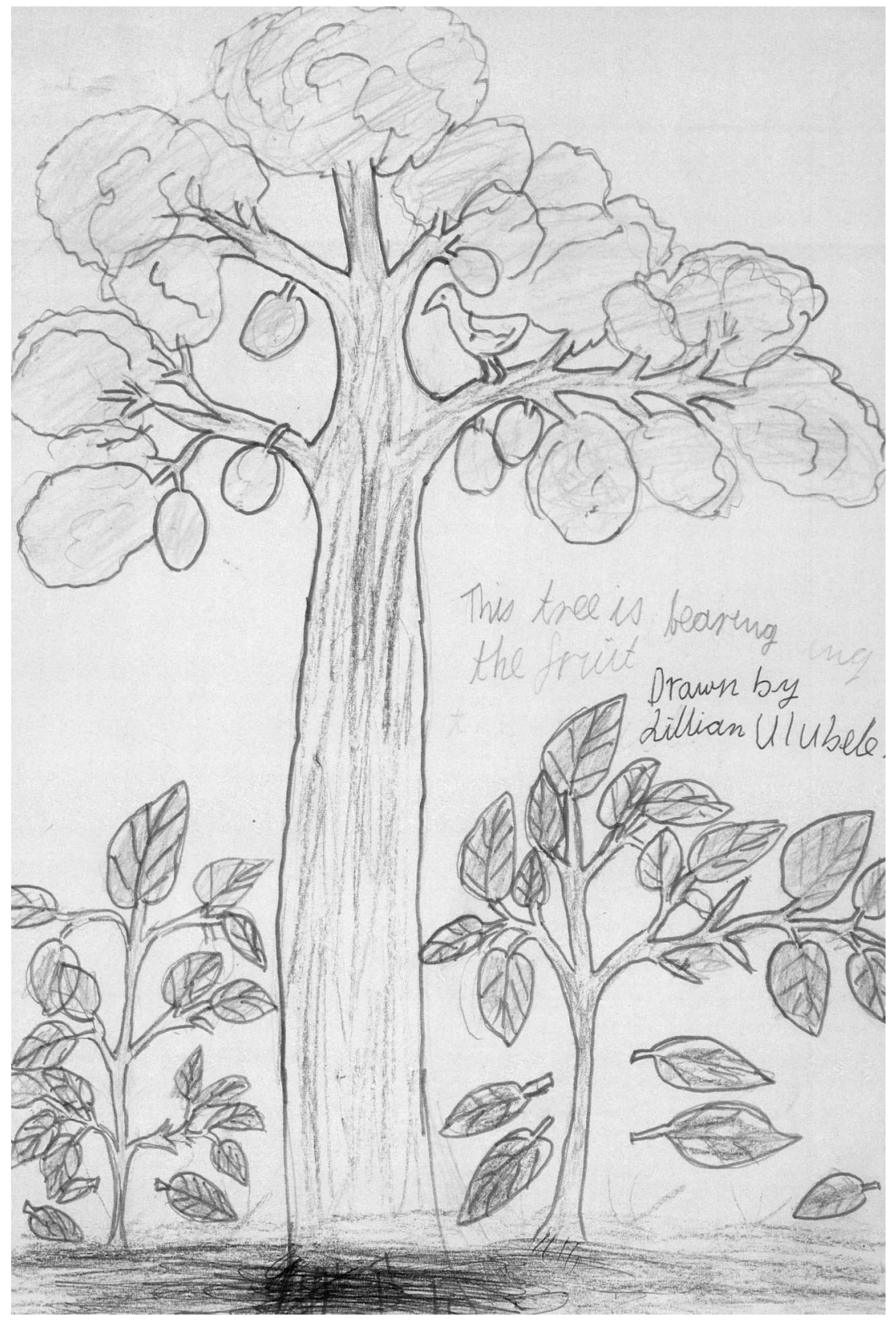

FIG. 7. - Dessin de Lillian Vlubele recueilli par Michel Legrand-Borderune. 